\title{
Preoperative neutrophil to lymphocyte ratio and platelet to lymphocyte ratio are associated with major adverse cardiovascular and cerebrovascular events in coronary heart disease patients undergoing non-cardiac surgery
}

Jan Larmann ${ }^{1 *}$ D, Jessica Handke1, Anna S. Scholz', Sarah Dehne', Christoph Arens', Hans-Jörg Gillmann², Florian Uhle ${ }^{1}$, Johann Motsch', Markus A. Weigand ${ }^{1}$ and Henrike Janssen ${ }^{1}$

\begin{abstract}
Background: Preoperative risk prediction in patients at elevated cardiovascular risk shows limited accuracy. Platelet to lymphocyte ratio (PLR) and neutrophil to lymphocyte ratio (NLR) indicate systemic inflammation. Both have been investigated for outcome prediction in the field of oncology and cardiovascular medicine, as well as risk prediction of adverse cardiovascular events in non-surgical patients at increased cardiovascular risk.

Methods: For this post-hoc analysis, we included all 38 coronary heart disease patients from the Leukocytes and Cardiovascular Perioperative Events cohort-1 study scheduled for elective non-cardiac surgery. We evaluated preoperative differential blood counts for association with major adverse cardiovascular and cerebrovascular events (MACCE) defined as the composite endpoint of death, myocardial ischemia, myocardial infarction, myocardial injury after non-cardiac surgery, or embolic or thrombotic stroke within 30 days after surgery. We used Youden's index to calculate cut-off values for PLR and NLR. Additive risk-predictive values were assessed using receiver operating characteristic curve and net reclassification (NRI) improvement analyses.
\end{abstract}

Results: Patients with the composite endpoint MACCE had higher PLR and NLR (309 [206; 380] vs. 160 [132; 203], $p=0.001 ; 4.9$ [3.5; 8.1] vs. 2.6 [2.2; 3.4]), $p=0.001$ ). Calculated cut-offs for PLR $>204.4$ and NLR $>3.1$ were associated with increased risk of 30-day MACCE (OR 7, 95\% Cl [1.2; 44.7], $p=0.034 ;$ OR 36, 95\% Cl [1.8; 686.6], $p=0.001$ ). Furthermore, NLR improved risk prediction in coronary heart disease patients undergoing non-cardiac surgery when combined with hs-cTnT or NT-proBNP (NRI total $=0.23, p=0.008, \mathrm{NRI}$ total $=0.26, p=0.005)$.

\footnotetext{
* Correspondence: jan.larmann@med.uni-heidelberg.de

'Department of Anaesthesiology, Heidelberg University Hospital, Im Neuenheimer Feld 110, 69120 Heidelberg, Germany

Full list of author information is available at the end of the article
}

C C The Author(s). 2020 Open Access This article is licensed under a Creative Commons Attribution 4.0 International License, which permits use, sharing, adaptation, distribution and reproduction in any medium or format, as long as you give appropriate credit to the original author(s) and the source, provide a link to the Creative Commons licence, and indicate if changes were made. The images or other third party material in this article are included in the article's Creative Commons licence, unless indicated otherwise in a credit line to the material. If material is not included in the article's Creative Commons licence and your intended use is not permitted by statutory regulation or exceeds the permitted use, you will need to obtain permission directly from the copyright holder. To view a copy of this licence, visit http://creativecommons.org/licenses/by/4.0/ The Creative Commons Public Domain Dedication waiver (http://creativecommons.org/publicdomain/zero/1.0/) applies to the data made available in this article, unless otherwise stated in a credit line to the data. 
(Continued from previous page)

Conclusions: Both PLR and NLR were associated with perioperative cardiovascular adverse events in coronary heart disease patients. NLR proved to be of additional value for preoperative risk stratification. Both PLR and NLR could be used as inexpensive and broadly available tools for perioperative risk assessment.

Trial registration: NCT02874508, August 22, 2016.

Keywords: Coronary heart disease, Perioperative care, Blood cell count, Platelet to lymphocyte ratio, Neutrophil to lymphocyte ratio

\section{Background}

Indices between cell populations from differential blood count (DBC), which is often routinely performed in the clinical setting, are a valuable option to gain further information on systemic inflammation and have been of interest within different fields of medical research. Risk prediction by the neutrophil to lymphocyte ratio (NLR) and platelet to lymphocyte ratio (PLR) was initially studied in the field of oncology $[1,2]$, followed by a vast amount of studies on the predictive value of NLR and PLR in cardiovascular medicine [3-5] and in a general population over the age of 45 [6].

In recent years, investigators have also focused on the perioperative period as well. Both indices are associated with postoperative acute kidney injury [7, 8]. Elevated preoperative NLR is associated with mortality and adverse outcome after cardiovascular surgery $[9,10]$, as well as with myocardial injury after non-cardiac surgery $[11,12]$ and lower long-term remission of type 2 diabetes after metabolic surgery [13]. PLR is associated with all-cause complications after abdominal aortic aneurysm repair [14].

However, there is only limited data on the predictive value for cardiovascular events in the large group on non-cardiac surgery patients. First, it is unknown whether elevated PLR is associated with cardiovascular adverse events in non-cardiac surgery patients. Second, neither NLR nor PLR have been evaluated in coronary heart disease-patients undergoing non-cardiac surgery who face higher rates of perioperative cardiovascular complications with challenging and inferior risk prediction [15-18]. Third,it is unknown if NLR or PLR have additive value for biomarker based preoperative prediction of perioperative cardiovascular events.

Therefore, in this post-hoc analysis, we investigated if preoperative cell subset counts from a conventional DBC, as well as PLR or NLR, are associated with the composite endpoint major adverse cardiovascular and cerebrovascular events (MACCE) within the first 30 postoperative days in coronary heart disease patients undergoing non-cardiac surgery. In addition, we tested the additive value for prediction of cardiovascular events in coronary heart disease patients undergoing non- cardiac surgery when conventional risk-prediction is complemented by NLR and PLR.

\section{Methods \\ Study population}

Data from the Leukocytes and Cardiovascular Perioperative Events cohort-1 (LeukoCAPE-1) study were used to conduct a post-hoc analysis. LeukoCAPE-1 was a singlecentre, prospective, observational cohort study conducted at the Department of Anaesthesiology, University Hospital Heidelberg, Heidelberg, Germany [17]. In summary, a total of 44 coronary heart disease patients scheduled for elective, non-cardiac surgery was screened for eligibility. Of those, 40 patients were successfully enrolled into the study. During follow-up, two patients withdrew consent and were excluded, resulting in a final study population of 38 participants. The main clinical and demographical baseline characteristics are published elsewhere [18]. In brief, the majority of patients were male, over the age of 60 years and classified as American Society of Anaesthesiologists physical status (ASA) $\geq 3$. Patients in the MACCE group more frequently suffered from preoperative diagnosed renal failure (Kidney Disease: Improving Global Outcomes stage $\geq 3$ ) and atrial fibrillation. Perioperative medication reflected patients' elevated cardiovascular risk profile. Patients who suffered MACCE took angiotensin converting enzyme inhibitors more frequently. The incidence of a history of percutaneous coronary intervention was $29 \%$ in the MACCE and $42 \%$ in the no MACCE group. $29 \%$ of patients in the MACCE group reported a history of myocardial infarction (MI) compared to $48 \%$ in the no MACCE group [18]. The trial was registered prior to patient enrolment at clinicaltrials.gov (NCT02874508, Date of registration: August 22, 2016). The study protocol conformed to the principles of the Declaration of Helsinki and the World Medical Association. After approval by the local Ethics Committee of the medical faculty of the Ruprecht-Karls University Heidelberg (S-351/ 2016, 4th August 2016), and after informed written consent, we enrolled consecutive general, vascular and urological surgery patients with documented coronary heart disease undergoing elective, in-patient, non-cardiac 
surgery between August and October 2016. We excluded patients younger than 18 years, pregnant or breastfeeding, and individuals with leukaemia, leukocytosis $(>10$ $\left.\mathrm{nll}^{-1}\right)$, or leukopenia $\left(<4 \mathrm{nll}^{-1}\right)$. Further exclusion criteria were emergency surgery, history of organ transplantation or splenectomy, immunosuppression, chemotherapy, GM-CSF or cortisone treatment less than 14 days prior to surgery, an intraoperative dexamethasone administration, or the occurrence of myocardial ischemia, MI, embolic or thrombotic stroke, congestive heart failure or serious cardiac arrhythmia within the past 28 days before enrolment.

\section{Data collection and conventional risk assessment}

After enrolment, we recorded previous cardiovascular and cerebrovascular events, demographic data, ASA classification, smoking status and current medication. A 12lead electrocardiogram was recorded preoperatively. Conventional risk assessment was based on revised cardiac risk index. Patients with high-sensitive cardiac Troponin $\mathrm{T}$ (hs-cTnT) $\geq 14 \mathrm{ngl}^{-1}$ or $\mathrm{N}$-terminal proBtype natriuretic peptide (NT-proBNP) $\geq 300 \mathrm{ngl}^{-1}$ were considered as at risk for perioperative MACCE [19].

\section{Sample collection and laboratory measurements}

As previously stated, blood samples were collected in the operating room prior to skin incision in EDTA for DBC and heparin tubes for biomarkers (Sarstedt, Nümbrecht, Germany) [17]. Samples were sent to the central laboratory within $30 \mathrm{~min}$, and analyses were performed according to standard operating procedures. NT-proBNP was measured preoperatively (Immulite, Siemens Healthcare Diagnostics, Erlangen, Germany); hs-cTnT was determined preoperatively and on postoperative days 1 to 3 (Cobas E4111, Roche Diagnostics, Mannheim, Germany). Automated DBC were performed in the central laboratory.

\section{Outcome analysis}

MACCE was defined as the composite endpoint of death, myocardial ischemia, MI, MINS, or embolic or thrombotic stroke within 30 days after surgery. In the LeukoCAPE-1 study a total of 7 patients reached this definition of the composite endpoint MACCE [18].

MINS [20] was defined as any raise in postoperative hs-cTnT $\geq 50 \mathrm{ngl}^{-1}$ [21] judged due to myocardial ischemia. Raising hs-cTnT was defined as an increase of at least 50\% from baseline [22]. Other secondary endpoints were individual components of the primary endpoint MACCE, new-onset atrial fibrillation, peripheral vascular occlusion and acute kidney injury. For outcome analysis, a postoperative 12-lead electrocardiogram was recorded on the third postoperative day, patient charts were screened and, if discharged prior to postoperative day
30, patients or their family doctors participated in a scripted telephone interview at the end of follow-up.

\section{Statistical analysis}

Not all blood cells were normally distributed. The MannWhitney U-test was chosen to compare all quantitative outcome data because it does not require any distributional assumption. Continuous data are presented as median [interquartile ranges (IQR)], unless otherwise stated. Boxes mark IQRs, whiskers extent from the 5th to 95th percentiles, and outliers are plotted as individual data points. Patients were categorized for the occurrence of MACCE. Two-sided $p$ values $<0.05$ were considered significant. To account for multiple comparisons, statistical analyses of preoperative leukocyte subset counts from DBC, as well as PLR and NLR were adjusted according to Bonferroni $(\alpha<0.05 / 8)$. If patients were discharged early, hs-cTnT data were imputed (last observation carried forward analysis). Receiver operating characteristic (ROC) analyses were performed to evaluate the discriminatory power of PLR and NLR in association with MACCE. The optimal threshold was calculated based on the maximized Youden index. Odds ratios (OR) [95\% confidence intervals $(\mathrm{CI})$ ] were calculated using Woolf and Baptista-Pike method as appropriate. Outcomes for ORs were all entities of the composite endpoint MACCE (death, myocardial ischemia, MI, MINS, or embolic or thrombotic stroke within 30 days after surgery), as well as all other secondary outcomes (new-onset atrial fibrillation, peripheral vascular occlusion and acute kidney injury). PLR and NLR cut-offs were used as independent variables. Net reclassification improvement (NRI) was performed to compare reclassification benefits and accuracies of preoperative PLR and NLR in addition to hs-cTnT or NT-proBNP for association with MACCE. The sample size $(n=40)$ for the LeukoCAPE-1 study was initially calculated to test the association of different leukocyte population counts with a surgical intervention [17]. Data from all 38 patients was included in this post-hoc analysis.

IBM SPSS Statistics 24.0 (SPSS, Chicago, USA), MedCalc 16.8 (MedCalc Software, Ostende, Belgium) and Prism 7.02 (GraphPad Prism Software, Inc., San Diego, US) were used for statistical analyses.

\section{Results \\ Preoperatively conducted differential blood counts and calculated ratios}

Results from DBC were recorded prospectively. Posthoc, we calculated patients' PLR and NLR after stratification for MACCE. After adjustment for multiple comparisons (Bonferroni $(\alpha<0.05 / 8=0.006)$ ), numbers of leukocyte subpopulations and platelets did not differ (Fig. 1 a-f). Patients suffering MACCE had higher PLR 
(309 [206; 380] vs. 160 [132; 203], $p=0.001)$ (Fig. 1 g) and higher NLR than patients without MACCE (4.9 [3.5; 8.1] vs. 2.6 [2.2; 3.4], $\mathrm{p}=0.001$ for MACCE vs. no MACCE) (Fig. 1 h).

Receiver operating characteristics analysis of NLR and PLR ROC curve analysis demonstrated that PLR had a high discriminatory value for MACCE $(\mathrm{AUC}=0.88 ; 95 \% \mathrm{CI}[0.75$;
$1], p=0.002)$. Based on maximized Youden index, the optimal cut-off was calculated to be $>204.4$ with a sensitivity of $86 \%$ and specificity of $77 \%$. The accuracy of the test was $82 \%$ (Fig. 2 a). Also, preoperative NLR had a high discriminatory ability for MACCE in our cohort (AUC $=0.88$; $95 \%$ CI $[0.78 ; 1], p=0.002)$. The optimal cut-off was calculated to be $>3.1$ with a sensitivity of $100 \%$ and specificity of $71 \%$. The accuracy of the test reached $82 \%$ (Fig. 2 b).
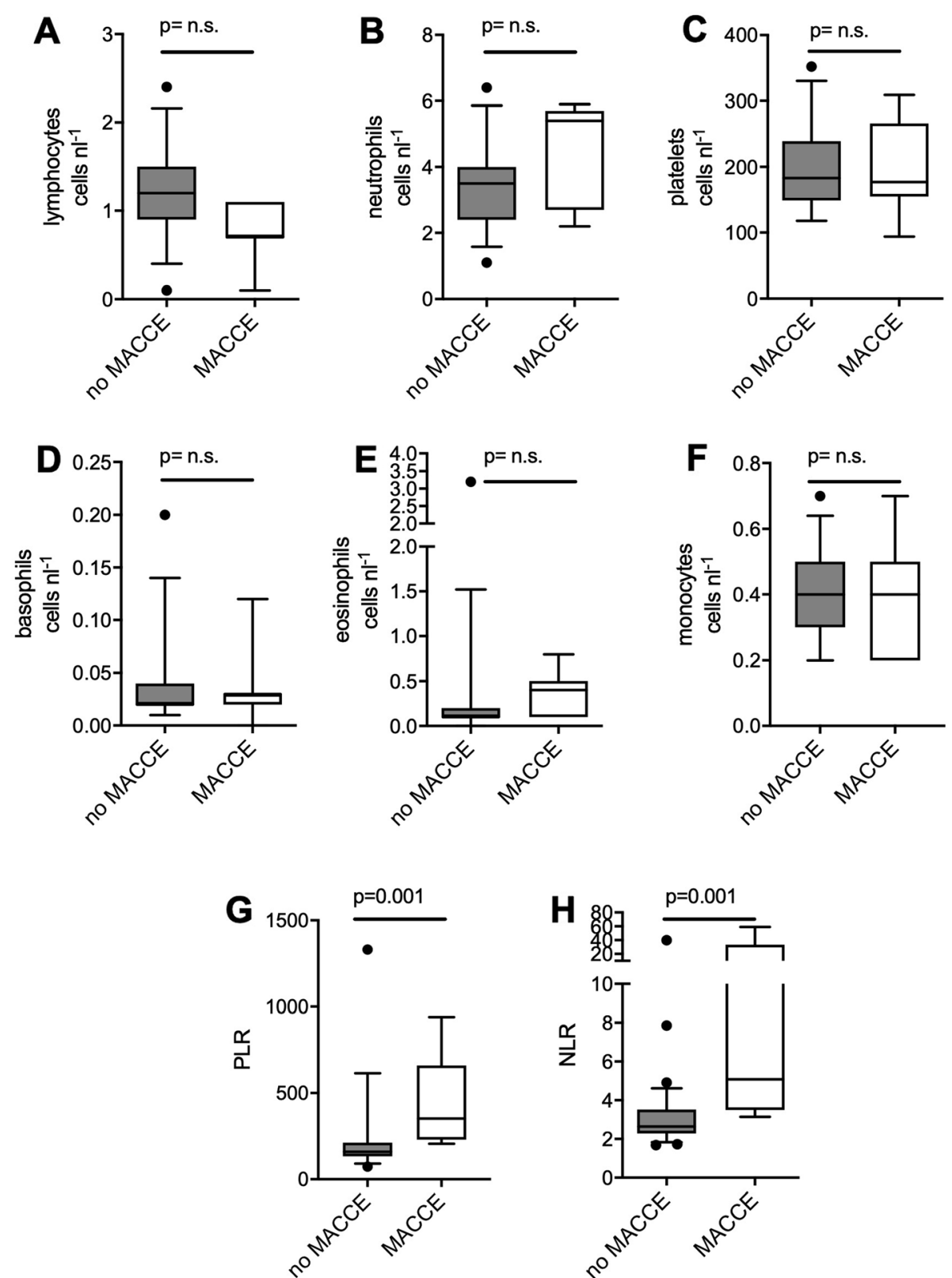

Fig. 1 Differential blood count. Prior to non-cardiac surgery, differential blood counts were performed in the 38 included patients. a-f After adjustment for multiple comparisons $(p=0.05 / 8=0.006)$, no difference in subpopulations could be detected between patients who suffered MACCE and patients who did not. $\mathbf{g}$, h Patients with MACCE had a higher PLR (309 [206; 380] vs. 160 [132; 203], $p=0.001)$, as well as higher NLR $(4.9[3.5 ; 8.1]$ vs. $2.6[2.2 ; 3.4], p=0.001)$ prior to surgery. MACCE: major adverse cardiovascular and cerebrovascular events, PLR: Platelet to Lymphocyte Ratio, NLR: Neutrophil to Lymphocyte Ratio 
Odds ratios for primary and secondary outcomes A preoperative PLR of $>204.4$ was associated with an increased risk of 30-day MACCE $(\mathrm{OR}=7 ; 95 \%$ CI [1.2; 44.7], $p=0.034$ ), but was not associated with any secondary outcome (Fig. 3 a). A preoperative NLR > 3.1 was associated with an increased risk of 30-day MACCE $(\mathrm{OR}=36 ; 95 \% \mathrm{CI}[1.8 ; 686.6], p=0.001)$. There was no association with any secondary outcome (Fig. 3 b).

\section{Additive risk predictive value of PLR or NLR to hs-cTnT T or NT-proBNP}

Addition of PLR to hs-cTnT let to improvement in the group without MACCE by correct reclassification of nine patients in the "not at risk"-group (NRI no MACCE = $3.00, p=0.003)$. In the group with MACCE, two patients were wrongly reclassified in the "not at risk"-group (NRI
MACCE $=-1.41, p=0.157)$. PLR did not prove to be of overall additional benefit for reclassification (NRI total $=$ 0.01, $p=0.984$ ) (Fig. 4 a). Addition of PLR to NT-proBNP also let to improvement in the group without MACCE by reclassifying eleven patients (NRI no MACCE $=3.32, p=0.001$ ). However, two patients were also wrongly reclassified in the "not at risk"-group (NRI MACCE $=-1.41, p=0.157)$. PLR did not prove to be of overall additional benefit for reclassification in combination with NT-proBNP (NRI total $=0.07, p=$ 0.762) (Fig. 4 b). NRI analysis demonstrated that the combination of hs-cTnT and NLR improved risk stratification with overall increased reclassification accuracy (NRI total $=0.23, p=$ 0.008) (Fig. 4 c). The addition of NLR to NT-proBNP also improved overall stratification (NRI total $=0.26, p=0.005$ ) (Fig. 4 d). For both hs-cTnT and NT-pro-BNP-based risk stratification, NLR reduced false positive classifications in the group without

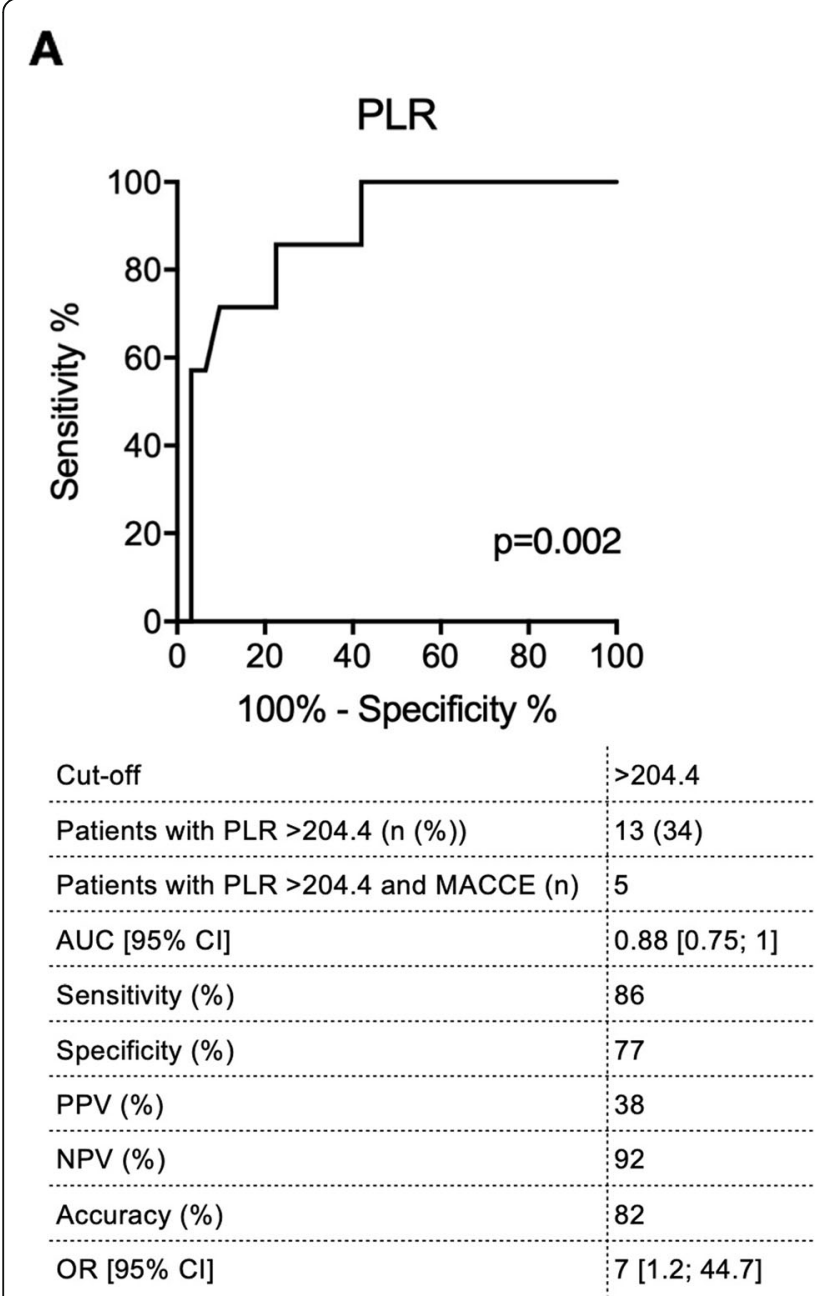

B

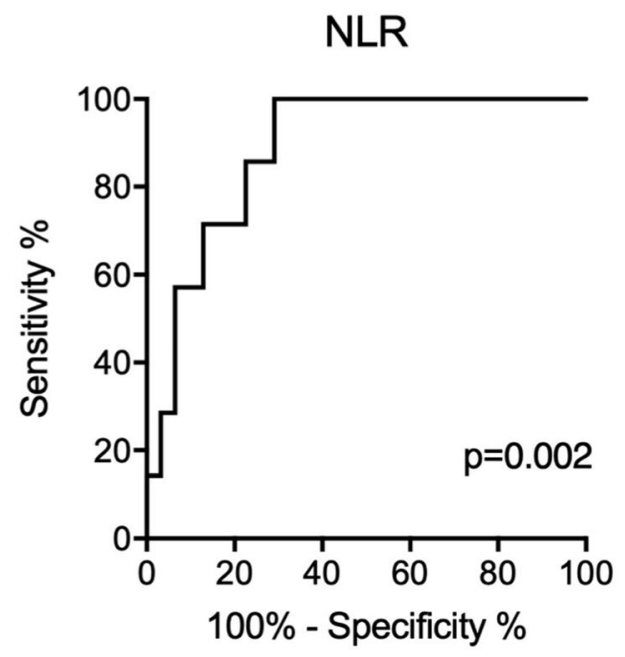

\begin{tabular}{|c|c|}
\hline Cut-off & $>3.1$ \\
\hline Patients with NLR >3.1(n (\%)) & $16(42)$ \\
\hline Patients with NLR >3.1 and MACCE (n) & 7 \\
\hline AUC $[95 \% \mathrm{Cl}]$ & $0.88[0.78 ; 1]$ \\
\hline Sensitivity (\%) & 100 \\
\hline Specificity (\%) & 71 \\
\hline PPV (\%) & 44 \\
\hline NPV (\%) & 100 \\
\hline Accuracy (\%) & 82 \\
\hline OR $[95 \% \mathrm{Cl}]$ & $36[1.8 ; 686.6]$ \\
\hline
\end{tabular}

Fig. 2 Receiver operating characteristics analysis of NLR and PLR. $\mathbf{a}, \mathbf{b}$ ) Receiver operating characteristics curve analyses were performed to evaluate discriminatory power of PLR and NLR for MACCE. Based on the maximized Youden Index, the optimal cut-off of PLR $>204.4$ and NLR > 3.1 were calculated. Test characteristics based on Woolf Chi-square analyses are displayed. PLR: Platelet to Lymphocyte Ratio, NLR: Neutrophil to Lymphocyte Ratio, MACCE: major adverse cardiovascular and cerebrovascular events, AUC: Area under the curve, Cl: confidence interval, PPV: positive predictive value, NPV: negative predictive value, OR: odds ratio 


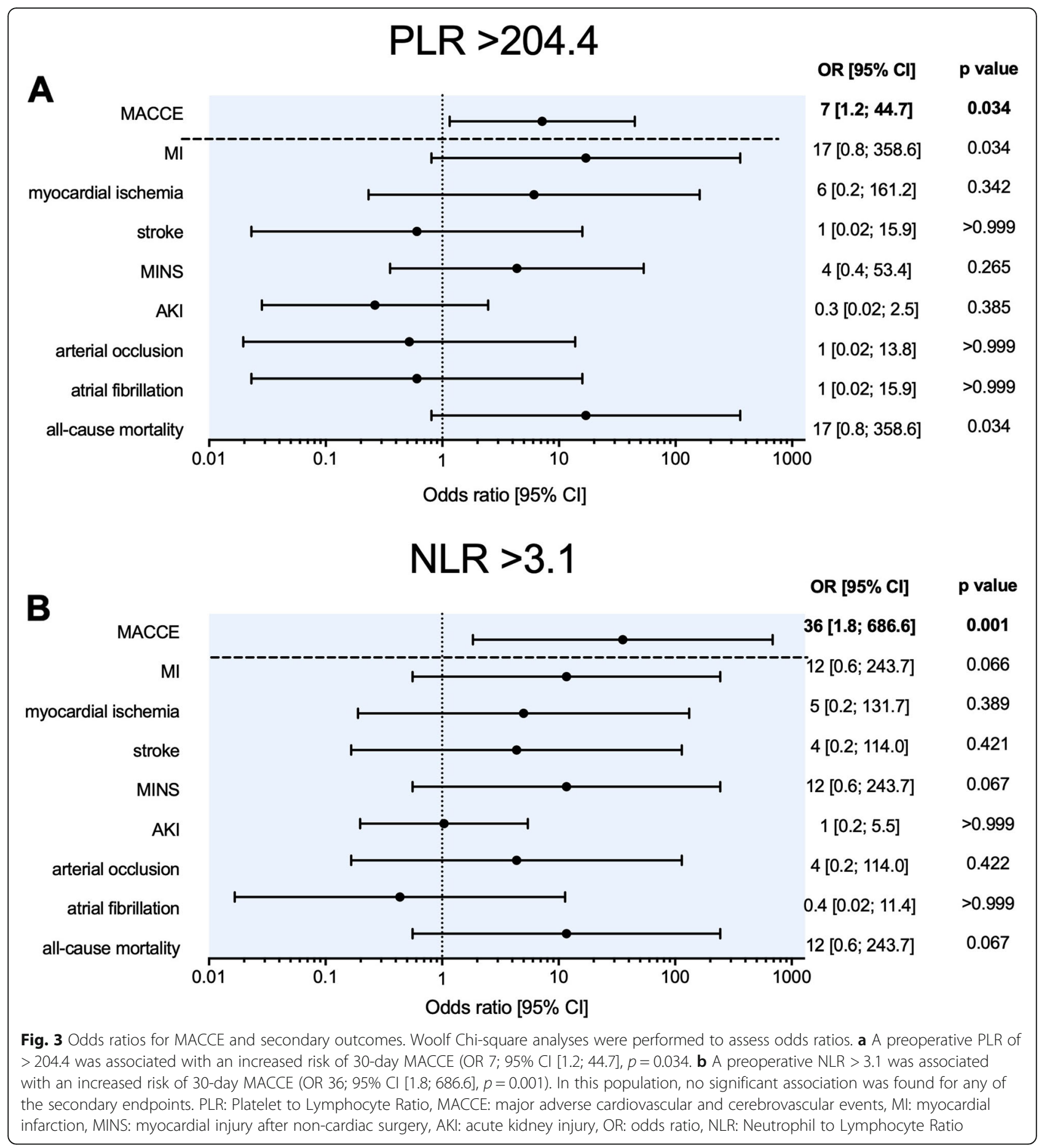

MACCE (hs-cTnT: NRI no MACCE $=2.65, p=0.008$; NTproBNP: NRI no MACCE $=2.83, p=0.005)$ (Fig. $4 \mathrm{c}, \mathrm{d}$ ).

\section{Discussion}

In this post-hoc analysis of the LeukoCAPE-1 study, we show that high preoperative PLR and NLR exceeding calculated cut-offs might be associated with MACCE within 30 days after non-cardiac surgery in coronary heart disease patients. The addition of NLR to the established biomarkers hs-cTnT or NT-proBNP improved preoperative risk prediction for MACCE in this population. Leukocytes play a causal role in the pathogenesis of atherosclerosis and misbalances are part of the mechanisms of plaque progression, destabilization, erosion or rupture, leading to myocardial injury, MI and stroke [23]. 


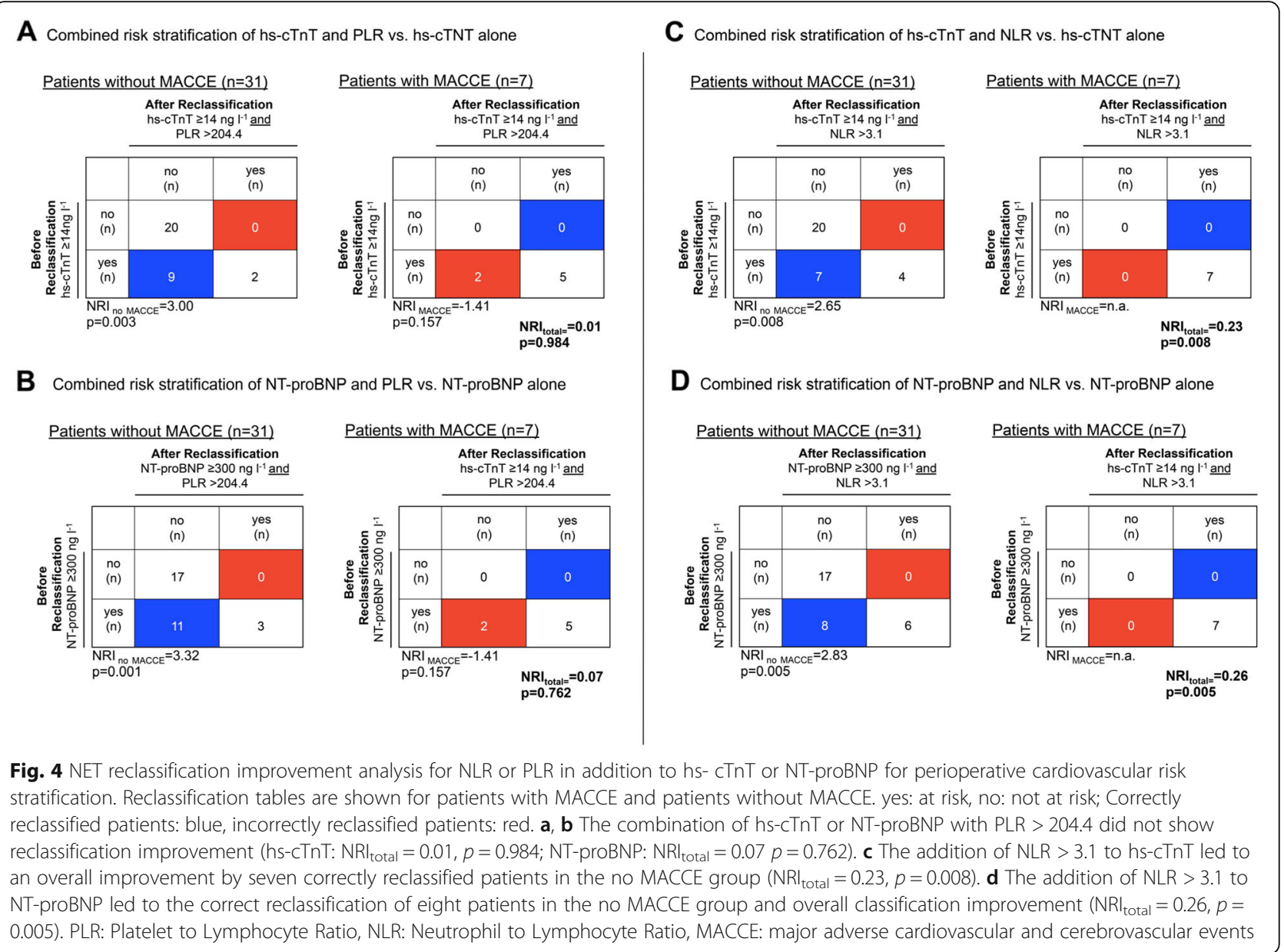

After necessary adjustment for multiple comparisons, we could not detect any differences in blood cell subpopulations in our cohort other than in NLR and in PLR. Due to physiological and pathological changes of absolute counts, there is an argument for a higher stability of hematological indices [24]. Moreover, both PLR and NLR present as a ratio of two inversely directed mechanisms that exacerbate atherosclerosis and cardiovascular events [24]. In line with our finding that PLR is associated with adverse cardiovascular outcome after non-cardiac surgery in coronary heart disease patients, Azab et al. demonstrated that PLR is associated with mortality after MI, independently of lymphocyte or platelet counts [24]. We calculated an ideal cut-off for PLR at 204.4 based on Youden-index which was associated with MACCE. Compared to the recent literature, our cut-off ranks high. It has been shown that preoperative PLR $>153.6$ predicts acute coronary syndrome in patients after carotid endarterectomy within an 18month follow-up period [25], and extreme values of < 91.5 or $>163.3$ are associated with complications after abdominal aortic aneurysm repair [26]. PLR was not associated with individual outcomes of the composite endpoint MACCE or any of the secondary outcomes analyzed in LeukoCAPE-1. As this analysis was conducted post-hoc, and the sample size was given, the study is underpowered to assess several secondary outcomes.

Our finding, that NLR is associated with adverse cardiovascular outcome, and improves risk prediction in coronary heart disease patients undergoing non-cardiac surgery, is in line with previous reports. In a cohort of patients at elevated cardiovascular risk, NLR is of predictive value for death or MI, even when DBC is within the normal range [27], and has proven to be a stronger independent predictor of mortality than other leukocyte subpopulations after MI [28]. NLR, but not DBC, predicts 2-year mortality after major vascular surgery [29]. Patients included in the LeukoCAPE-1 study underwent elective surgery. Correspondingly, median neutrophil count of 3.5 in our cohort is far lower than in patients undergoing emergency surgery [30], but also surprisingly low for patients with underlying chronic vascular inflammation [31].

Recently, two meta-anaylses have supported that high NLR on hospital admission of patients suffering MI is associated with deleterious outcome, including long-term 
mortality and adverse cardiovascular events [32, 33]. This extends to stroke, as high NLR is correlated with increased risk and worse functional outcome after ischemic stroke and with higher mortality after ischemic, as well as hemorrhagic stroke [34-36]. The majority of patients included in these studies have preexisting chronic cardiovascular disease. We could not detect any association with MI or stroke in our cohort, probably because the study is underpowered to test the secondary outcomes.

Patients with coronary heart disease are underrepresented in previous studies in the perioperative field [12]. Therefore, our cohort of patients with preoperatively diagnosed coronary heart disease is of particular interest for further studies into NLR as a predictive marker of cardiovascular events, especially as performance of current risk predicting tools is limited in this cohort [15]. Our post-hoc analysis showed that the inexpensive addition of NLR to the widely recommended biomarkers hs-cTnT or NT-proBNP $[19,37]$ improved risk classification mainly by reclassifying patients from "false positive" to "correct negative" which may lead to better resource management by reducing the number of patients stratified as at risk for MACCE.

Our study has several limitations. The analysis was performed post-hoc, rendering results explorative rather than confirmative. However, the results are hypothesisgenerating and should be investigated in a larger prospective observational study in patients at elevated risk for perioperative cardiovascular complications. The number of patients is relatively low as it was originally calculated for testing the association of non-cardiac surgery with changes in counts of leukocyte subpopulation [17]. Confidence intervals for both NLR and PLR are wide and likely related to small sample size. However, the effect estimates not only for the primary endpoint MACCE, but also for other secondary cardiovascular endpoints such as MI, myocardial ischemia, MINS and all-cause mortality were $\geq 4$ and can serve in the design of future clinical trials. Because of the limited number of patients, this study is insufficient for multivariate and logistic regression analyses. Future studies are needed to test whether NLR and PLR independently predict MACCE in patients at elevated risk for cardiovascular events.

\section{Conclusions}

The findings of our post-hoc analysis suggest that both PLR > 204.4 and NLR > 3.1 are associated with perioperative 30-day MACCE in coronary heart disease patients. The addition of NLR to established biomarkers for cardiovascular risk stratification improved prediction in patients at elevated risk, potentially leading to improved resource management in perioperative care. PLR, as well as NLR are inexpensive, readily available markers.
Preoperative PLR and NLR might be calculated to allow perioperative caregivers a more accurate prediction of perioperative cardiovascular events in coronary heart disease patients undergoing non-cardiac surgery.

\begin{abstract}
Abbreviations
ASA: American society of anaesthesiologists physical status; Cl: Confidence interval; DBC: Differential blood count; Hs-cTnT: High-sensitive cardiac troponin T; IQR: Interquartile ranges; LeukoCAPE-1: Leukocytes and Cardiovascular Perioperative Events cohort-1; MACCE: Major adverse cardiovascular and cerebrovascular events; Ml: Myocardial infarction; MINS: Myocardial injury after non-cardiac surgery; NLR: Neutrophil to lymphocyte ratio; NRI: Net reclassification improvement; NT-proBNP: Nterminal proB-type natriuretic peptide; OR: Odds ratio; PLR: Platelet to lymphocyte ratio; ROC: Receiver operating characteristic
\end{abstract}

\section{Acknowledgements}

Not applicable.

\section{Authors' contributions}

$J \mathrm{~L}, J H, A S S, J M$ and $H J$ designed and planned the study; JL, SD, CA and HJ screened and included patients; JH, SD, CA and ASS performed measurements; JL, JH, ASS, HJG, FU, MAW, JM and HJ analyzed and interpreted data; $\mathrm{J}$ and $\mathrm{HJ}$ wrote the manuscript; all authors read and approved the final manuscript.

\section{Funding}

This study was supported by departmental funds by the Department of Anaesthesiology, University Hospital Heidelberg, Heidelberg, Germany. The source of funding did not have any impact on study design; collection, analysis, and interpretation of data or writing of the manuscript.

\section{Availability of data and materials}

The datasets used and/or analyzed during the current study are available from the corresponding author on reasonable request.

\section{Ethics approval and consent to participate}

The study was registered prior to patient enrolment at clinicaltrials.gov (NCT02874508, Date of registration: August 22, 2016). The study protocol conformed to the principles of the Declaration of Helsinki and the World Medical Association. Patients were enrolled between August and October 2016 after approval by the local Ethics Committee of the medical faculty of the Ruprecht-Karls University Heidelberg (S-351/2016, 4th August 2016) and with patients' written informed consent.

Consent for publication

Not applicable.

\section{Competing interests}

The authors declare that they have no competing interests.

\section{Author details}

'Department of Anaesthesiology, Heidelberg University Hospital, Im Neuenheimer Feld 110, 69120 Heidelberg, Germany. ${ }^{2}$ Department of Anaesthesiology and Intensive Care Medicine, Hannover Medical School, Hannover, Germany.

Received: 2 August 2019 Accepted: 30 April 2020

Published online: 18 May 2020

\section{References}

1. Templeton AJ, McNamara MG, Seruga B, Vera-Badillo FE, Aneja P, Ocana A, et al. Prognostic role of neutrophil-to-lymphocyte ratio in solid tumors: a systematic review and meta-analysis. J Natl Cancer Inst. 2014;106(6):dju124.

2. Li B, Zhou P, Liu Y, Wei H, Yang X, Chen T, et al. Platelet-to-lymphocyte ratio in advanced Cancer: review and meta-analysis. Clin Chim Acta. 2018;483:4856.

3. Balta S, Celik T, Mikhailidis DP, Ozturk C, Demirkol S, Aparci M, et al. The relation between atherosclerosis and the neutrophil-lymphocyte ratio. Clin Appl Thromb Hemost. 2016;22(5):405-11. 
4. Kalay N, Dogdu O, Koc F, Yarlioglues M, Ardic I, Akpek M, et al. Hematologic parameters and angiographic progression of coronary atherosclerosis. Angiology. 2012;63(3):213-7.

5. Balta S, Ozturk C. The platelet-lymphocyte ratio: a simple, inexpensive and rapid prognostic marker for cardiovascular events. Platelets. 2015;26(7):6801.

6. Fest J, Ruiter TR, Groot Koerkamp B, Rizopoulos D, Ikram MA, van Eijck CHJ, et al. The neutrophil-to-lymphocyte ratio is associated with mortality in the general population: the Rotterdam study. Eur J Epidemiol. 2019;34(5):46370.

7. Parlar H, Saskin H. Are pre and postoperative platelet to lymphocyte ratio and neutrophil to lymphocyte ratio associated with early postoperative AKI following CABG? Braz J Cardiovasc Surg. 2018;33(3):233-41.

8. Chen D, Xiao D, Guo J, Chahan B, Wang Z. Neutrophil-lymphocyte count ratio as a diagnostic marker for acute kidney injury: a systematic review and meta-analysis. Clin Exp Nephrol. 2019. https://doi.org/10.1007/s10157-01901800-y.

9. Tan TP, Arekapudi A, Metha J, Prasad A, Venkatraghavan L. Neutrophillymphocyte ratio as predictor of mortality and morbidity in cardiovascular surgery: a systematic review. ANZ J Surg. 2015;85(6):414-9.

10. Silberman S, Abu-Yunis U, Tauber R, Shavit L, Grenader T, Fink D, et al. Neutrophil-lymphocyte ratio: prognostic impact in heart surgery. Early outcomes and late survival. Ann Thorac Surg. 2018;105(2):581-6.

11. Ackland GL, Abbott TEF, Cain D, Edwards MR, Sultan P, Karmali SN, et al. Preoperative systemic inflammation and perioperative myocardial injury: prospective observational multicentre cohort study of patients undergoing non-cardiac surgery. Br J Anaesth. 2019;122(2):180-7.

12. Durmus $\mathrm{G}$, Belen $\mathrm{E}$, Can MM. Increased neutrophil to lymphocyte ratio predicts myocardial injury in patients undergoing non-cardiac surgery. Heart Lung. 2018;47(3):243-7.

13. Bonaventura A, Liberale L, Carbone F, Vecchie A, Bonomi A, Scopinaro N, et al. Baseline neutrophil-to-lymphocyte ratio is associated with long-term T2D remission after metabolic surgery. Acta Diabetol. 2019;56(7):741-8.

14. Lareyre F, Carboni J, Chikande J, Massiot N, Voury-Pons A, Umbdenstock E, et al. Association of Platelet to lymphocyte ratio and risk of 30-day postoperative complications in patients undergoing abdominal aortic surgical repair. Vasc Endovasc Surg. 2019;53(1):5-11.

15. Ford MK, Beattie WS, Wijeysundera DN. Systematic review: prediction of perioperative cardiac complications and mortality by the revised cardiac risk index. Ann Intern Med. 2010;152(1):26-35.

16. Janssen H, Felgner L, Kummer L, Gillmann HJ, Schrimpf C, Rustum S, et al. Sequential surgical procedures in vascular surgery patients are associated with perioperative adverse cardiac events. Front Cardiovasc Med. 2020;7:13.

17. Handke J, Scholz AS, Gillmann HJ, Janssen H, Dehne S, Arens C, et al. Elevated presepsin is associated with perioperative major adverse cardiovascular and cerebrovascular complications in elevated-risk patients undergoing noncardiac surgery: the leukocytes and cardiovascular perioperative events study. Anesth Analg. 2018. https://doi.org/10.1213/ANE. 0000000000003738.

18. Scholz AS, Handke J, Gillmann HJ, Zhang Q, Dehne S, Janssen H, et al. Frontline science: low regulatory $T$ cells predict perioperative major adverse cardiovascular and cerebrovascular events after noncardiac surgery. J Leukoc Biol. 2019. https://doi.org/10.1002/JLB.5HI1018-392RR.

19. Duceppe E, Parlow J, MacDonald P, Lyons K, McMullen M, Srinathan S, et al. Canadian cardiovascular society guidelines on perioperative cardiac risk assessment and management for patients who undergo noncardiac surgery. Can J Cardiol. 2017;33(1):17-32.

20. Botto F, Alonso-Coello P, Chan MT, Villar JC, Xavier D, Srinathan S, et al. Myocardial injury after noncardiac surgery: a large, international, prospective cohort study establishing diagnostic criteria, characteristics, predictors, and 30-day outcomes. Anesthesiology. 2014;120(3):564-78.

21. Giannitsis E, Becker M, Kurz K, Hess G, Zdunek D, Katus HA. High-sensitivity cardiac troponin $\mathrm{T}$ for early prediction of evolving non-ST-segment elevation myocardial infarction in patients with suspected acute coronary syndrome and negative troponin results on admission. Clin Chem. 2010; 56(4):642-50

22. Alcock RF, Kouzios D, Naoum C, Hillis GS, Brieger DB. Perioperative myocardial necrosis in patients at high cardiovascular risk undergoing elective non-cardiac surgery. Heart. 2012;98(10):792-8.

23. Swirski FK, Nahrendorf M. Leukocyte behavior in atherosclerosis, myocardial infarction, and heart failure. Science. 2013;339(6116):161-6.
24. Azab B, Shah N, Akerman M, McGinn JT Jr. Value of platelet/lymphocyte ratio as a predictor of all-cause mortality after non-ST-elevation myocardial infarction. J Thromb Thrombolysis. 2012;34(3):326-34.

25. Bonaventura A, Carbone F, Liberale L, Mach F, Roth A, Burger F, et al. Platelet-to-lymphocyte ratio at the time of carotid endarterectomy is associated with acute coronary syndrome occurrence. J Cardiovasc Med (Hagerstown). 2020;21(1):80-2.

26. Lareyre F, Carboni J, Chikande J, Massiot N, Voury-Pons A, Umbdenstock E, et al. Association of Platelet to lymphocyte ratio and risk of 30-day postoperative complications in patients undergoing abdominal aortic surgical repair. Vasc Endovascular Surg. 2019;53(1):5-11.

27. Horne BD, Anderson JL, John JM, Weaver A, Bair TL, Jensen KR, et al. Which white blood cell subtypes predict increased cardiovascular risk? J Am Coll Cardiol. 2005;45(10):1638-43.

28. Azab B, Zaher M, Weiserbs KF, Torbey E, Lacossiere K, Gaddam S, et al. Usefulness of neutrophil to lymphocyte ratio in predicting short- and longterm mortality after non-ST-elevation myocardial infarction. Am J Cardiol. 2010;106(4):470-6.

29. Bhutta H, Agha R, Wong J, Tang TY, Wilson YG, Walsh SR. Neutrophillymphocyte ratio predicts medium-term survival following elective major vascular surgery: a cross-sectional study. Vasc Endovasc Surg. 2011;45(3): 227-31.

30. Vaughan-Shaw PG, Rees JR, King AT. Neutrophil lymphocyte ratio in outcome prediction after emergency abdominal surgery in the elderly. Int J Surg. 2012;10(3):157-62.

31. Groot HE, van Blokland IV, Lipsic E, Karper JC, van der Harst P. Leukocyte profiles across the cardiovascular disease continuum: a population-based cohort study. J Mol Cell Cardiol. 2019;138:158-64.

32. Dentali F, Nigro O, Squizzato A, Gianni M, Zuretti F, Grandi AM, et al. Impact of neutrophils to lymphocytes ratio on major clinical outcomes in patients with acute coronary syndromes: a systematic review and meta-analysis of the literature. Int J Cardiol. 2018;266:31-7.

33. Zhang S, Diao J, Qi C, Jin J, Li L, Gao X, et al. Predictive value of neutrophil to lymphocyte ratio in patients with acute ST segment elevation myocardial infarction after percutaneous coronary intervention: a meta-analysis. BMC Cardiovasc Disord. 2018;18(1):75.

34. Song SY, Zhao XX, Rajah G, Hua C, Kang RJ, Han YP, et al. Clinical significance of baseline neutrophil-to-lymphocyte ratio in patients with ischemic stroke or hemorrhagic stroke: an updated meta-analysis. Front Neurol. 2019;10:1032

35. Wang L, Song Q, Wang C, Wu S, Deng L, Li Y, et al. Neutrophil to lymphocyte ratio predicts poor outcomes after acute ischemic stroke: a cohort study and systematic review. J Neurol Sci. 2019;406:116445.

36. Giede-Jeppe A, Madzar D, Sembill JA, Sprugel MI, Atay S, Hoelter P, et al. Increased neutrophil-to-lymphocyte ratio is associated with unfavorable functional outcome in acute ischemic stroke. Neurocrit Care. 2019. https:// doi.org/10.1007/s12028-019-00859-5.

37. Kristensen SD, Knuuti J, Saraste A, Anker S, Botker HE, De Hert S, et al. 2014 ESC/ESA guidelines on non-cardiac surgery: cardiovascular assessment and management: the joint task force on non-cardiac surgery: cardiovascular assessment and management of the European Society of Cardiology (ESC) and the European Society of Anaesthesiology (ESA). Eur J Anaesthesiol. 2014;31(10):517-73.

\section{Publisher's Note}

Springer Nature remains neutral with regard to jurisdictional claims in published maps and institutional affiliations.

Ready to submit your research? Choose BMC and benefit from:

- fast, convenient online submission

- thorough peer review by experienced researchers in your field

- rapid publication on acceptance

- support for research data, including large and complex data types

- gold Open Access which fosters wider collaboration and increased citations

- maximum visibility for your research: over $100 \mathrm{M}$ website views per year

At $\mathrm{BMC}$, research is always in progress.

Learn more biomedcentral.com/submission 